\title{
Resistance Temperature Detector Sensor with a Copper Pattern on the Printed Circuit Board
}

\author{
Sang-Yoon Kim ${ }^{1,3}$, Jong-Dae Kim ${ }^{2,3}$, Yu-Seop Kim ${ }^{2,3}$ Hye-Jeong Song ${ }^{2,3}$ and \\ Chan-Young Park ${ }^{2,3^{*}}$ \\ ${ }^{1}$ Department of Computer Engineering, Hallym University, Korea \\ ${ }^{2}$ Department of Convergence Software, Hallym University, Korea \\ ${ }^{3}$ Bio-IT Research Center, Hallym University, Korea \\ \{lastamor, kimjd, yskim01, hjsong, cypark\}@hallym.ac.kr
}

\begin{abstract}
A biochip can obtain various types of genetic information over a short period of time and enable genetic analysis. A biochip needs temperature sensors to measure temperature. A resistance temperature detector temperature sensor chip, made of copper using a printed circuit board, was introduced in this study. A printed circuit board is readily usable for copper pattern design for the substrate of a biochip. To measure biochip resistance, we tested whether it was possible to accurately measure the temperature using resistances measured at room temperature and at 55, 72, 85, and $95^{\circ} \mathrm{C}$ in a water bath. However, in analyzing the results obtained, the printed circuit board based resistance temperature detector sensor was found to be inadequate for use as a biochip temperature sensor, although it showed potential for use as a general-purpose resistance temperature detector sensor.
\end{abstract}

Keywords: Resistance Temperature Detector, Metal Thin Film Sensor, PCB Pattern Resistance

\section{Introduction}

A biochip is a very useful tool for analyzing infinitesimal amounts of samples at superhigh speeds, for selectively and functionally fixing high-molecular biomaterials on the surfaces of chips, and for analyzing surface-bound biomaterials. The main technique used to analyze the data collected with biochips is polymerase chain reaction (PCR).

The PCR technique, invented by an American biochemist, Kary Mullis, in 1983, is a bimolecular technique that replicates and amplifies a very small amount of DNA (deoxyribonucleic acid) more than a hundredfold [1,2]. This technique plays a significant role in broad areas associated with handling DNA, such as biochemistry, molecular biology, medicine, forensic medicine for criminal investigation, and biological classification and analysis. PCR generally consists of the following three steps:

(1) DNA Denaturation: DNA is heated to $94^{\circ} \mathrm{C}$, and the double strands of the DNA are separated into single strands of DNA.

(2) Primer Annealing: Each of the two different primers is annealed to a single stranded DNA template separated after heating. at $55-65^{\circ} \mathrm{C}$.

(3) DNA Extension: At $72^{\circ} \mathrm{C}$, the primers are extended by DNA polymerase.

The principle of PCR is to perform these three steps repeatedly and thus amplify the target region of the DNA. In this procedure, Peltier and polyimide flexible heaters have been used to increase the temperature of the DNA, but the unit prices of such heaters are high. An earlier study was conducted by making a printed circuit board (PCB), which has a lower unit cost than the other materials mentioned, to serve as the substrate [3]. 
In addition, sensors are needed to accurately measure the proper temperature at each step. Temperature sensors such as thermocouple sensors, thermistors, and resistance temperature detectors (RTD) are installed in the biochip, on the surface where the DNA is amplified, for this purpose [4-8].

In this study, an RTD temperature sensor chip was manufactured using PCB as the substrate of biochip. The material used in the PCB was copper $(\mathrm{Cu})$, and it is easy to design a $\mathrm{Cu}$ pattern for this purpose. In addition, an RTD sensor is the most stable of the types of sensors that could be used, has good repeatability and reproducibility, and is suitable for measurement of the temperatures of sensitive thermal components. An RTD sensor works by sensing variations in electrical resistance with temperature, which occur in almost all materials. This variation is repeatable and reproducible in a certain material, which makes accurate measurement of temperature possible. To verify the accuracy of the PCB-based RTD temperature sensor, manufactured as described above, we tested whether it was possible to accurately determine temperatures from resistances measured at room temperature and at $55,72,85$, and $95^{\circ} \mathrm{C}$ in a water bath.

\section{Methods}

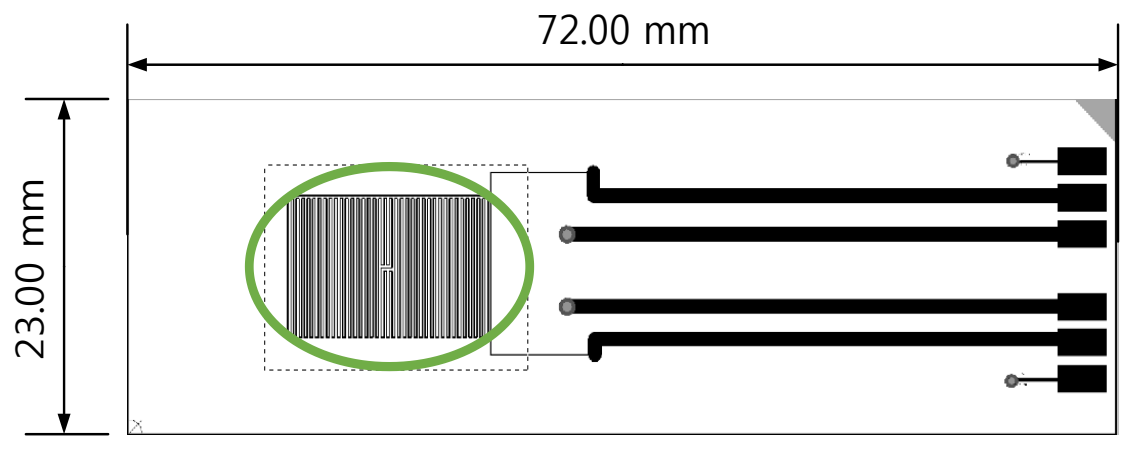

Figure 1. PCB Schematic

Figure 1 is a schematic of the PCB designed in this study, which was $72.0 \mathrm{~mm}$ in length and $23.0 \mathrm{~mm}$ in width. This chip did not undergo auto optical inspection (AOI). AOI can capture several millions of spots on the surface of a product and detect various defects, such as component defects, broken wires, a short circuit, marks or stains, and misplacement of components. However, the AOI process was not used in this study because it involves additional cost and thus could increase the unit price.

Figure 2 shows an enlargement of the area of the RTD sensor within the oval in Figure 1. This area is $15.0 \mathrm{~mm}$ in length and $10.0 \mathrm{~mm}$ in width.

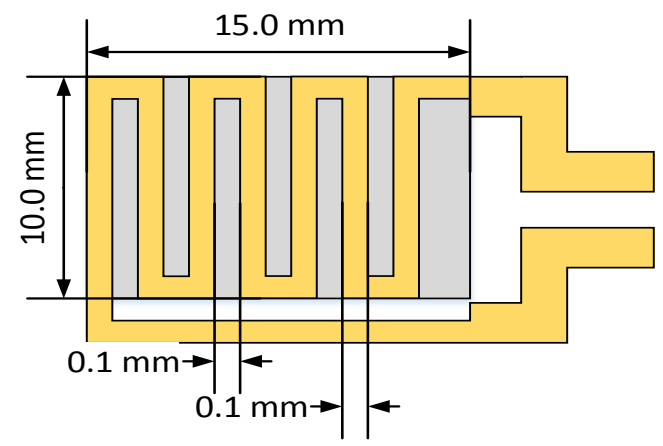

Figure 2. RTD Sensor Pattern 


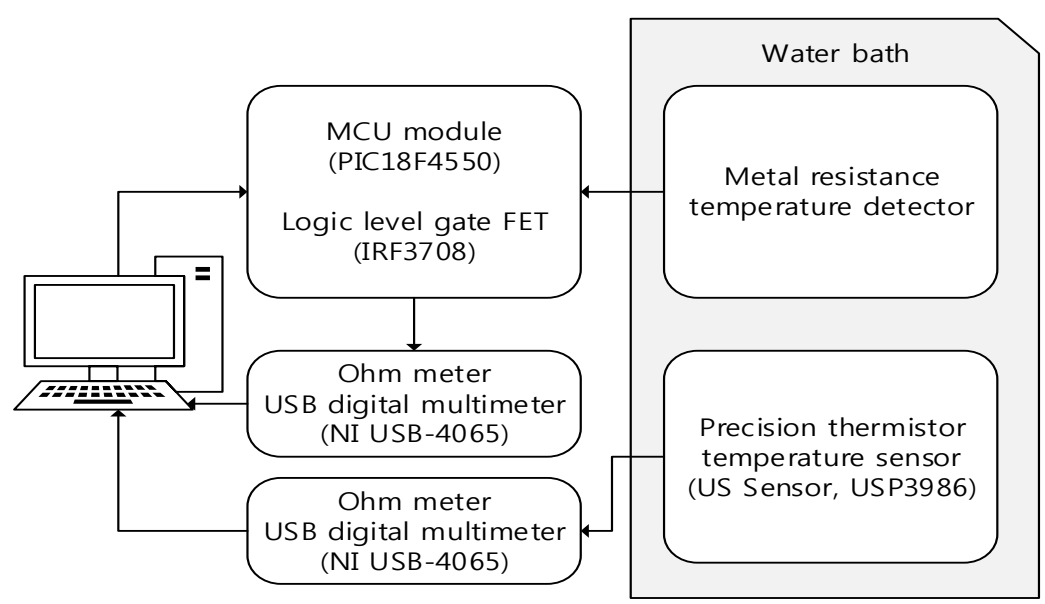

Figure 3. System Structure for Measuring the Resistance of a PCB-Based RTD Chip

The structure of the system that was designed and manufactured to measure RTD chip resistance is shown in Figure 3. Measurements were obtained using a water bath (HETO OBN 8 and temperature control unit HETO HMT 200) at temperatures of $20,55,70,85$, and $95^{\circ} \mathrm{C}$, according to the temperature range used to amplify DNA in PCR. Ten PCB-based RTD sensor chips fabricated according to the design shown in Figure 1 were placed in the water bath at each temperature. In addition, a precision thermistor temperature sensor (US Sensor, USP3986) was placed in the water bath to verify the accuracy of the temperature displayed for the water bath.

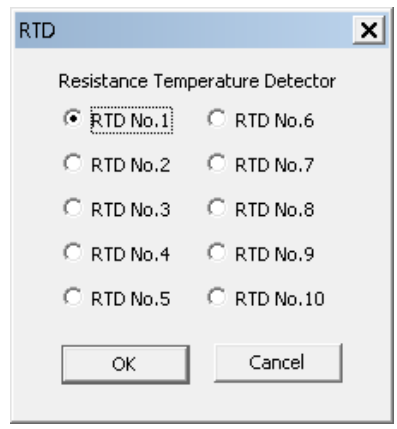

Figure 3. MFC (Microsoft Foundation Class) Program to Select an RTD Sensor

Each RTD sensor can be selected using a personal computer (PC)-based MFC program, as shown in Figure 4. The RTD sensor number selected is transferred to a micro controller unit (MCU). The MCU turns on the switches of a field effect transistor (FET), illustrated in Figure 5, based on the RTD sensor number that is transferred. One of the ten RTD sensors is connected to a universal serial bus (USB) digital multimeter (NI USB-4065), depending on the selected switch, and the resistance measured at each water temperature is transferred to the PC. In addition, the precision thermistor temperature sensor placed in the water bath measures the resistance through another USB digital multimeter. The resistance thus measured is displayed in a measurement program for the PC provided by NI (National Instruments).

The measurements displayed in the program immediately after RTD sensor selection using the MFC program were found to be very unstable. Thus, means of 
the maximum and minimum values were calculated and recorded once the variation in the resistance displayed had stabilized.

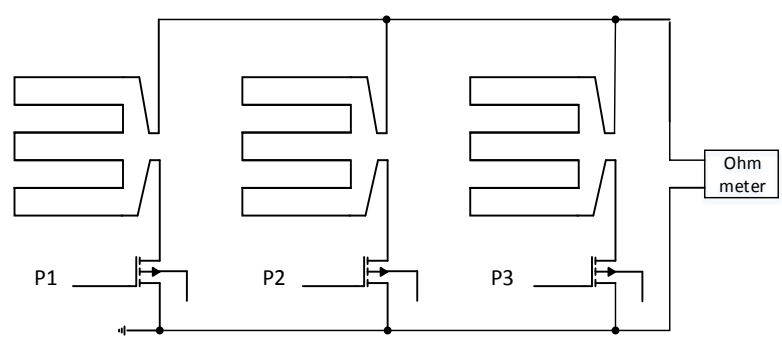

Figure 4. NTC Sensor Measuring Circuit Using a Logic Level Gate FET

Figure 5 illustrates the NTC sensor measuring circuit, which uses a logic level gate FET. An FET is a transistor that controls the current of the source and drain based on the principle of creation of a gate through which electrons or holes flow as a result of an electrical field applied to a channel connected to the voltage and gate electrodes. In other words, an FET is an electrical switch. A typical FET operates at a voltage of approximately $5 \mathrm{~V}$, but a logic level FET must be operated at a lower voltage, such as $3.3 \mathrm{~V}$. The reason for this is that the MCU port cannot provide a constant voltage of $5 \mathrm{~V}$.

In this study, a logic level FET was used to reduce the inconvenience of connecting individual RTD sensors to USB digital multimeters.

\section{Results}

The resistance values for the ten RTD sensor chips, measured at each set temperature, are shown in Table 1.

Table 1. Resistance of Ten RTD Sensors at Each Temperature

\begin{tabular}{ccccccc}
\hline $\begin{array}{c}\text { Chip } \\
\text { No }\end{array}$ & 20.6 & 55.0 & 72.0 & 85.0 & 95.0 & $\begin{array}{c}95- \\
55^{\circ} \mathrm{C}\end{array}$ \\
\hline 1 & 6.15 & 6.85 & 7.20 & 7.47 & 7.67 & 0.82 \\
2 & 5.95 & 6.63 & 6.97 & 7.23 & 7.42 & 0.79 \\
3 & 6.00 & 6.69 & 7.03 & 7.30 & 7.49 & 0.80 \\
4 & 5.27 & 5.86 & 6.16 & 6.38 & 6.55 & 0.69 \\
5 & 6.01 & 6.70 & 7.05 & 7.31 & 7.51 & 0.81 \\
6 & 5.05 & 5.62 & 5.90 & 6.12 & 6.28 & 0.66 \\
7 & 6.31 & 7.04 & 7.40 & 7.68 & 7.90 & 0.86 \\
8 & 5.19 & 5.77 & 6.06 & 6.28 & 6.45 & 0.68 \\
9 & 5.56 & 6.20 & 6.51 & 6.75 & 6.93 & 0.73 \\
10 & 5.39 & 6.00 & 6.29 & 6.52 & 6.70 & 0.70 \\
\hline
\end{tabular}

Table 1 shows that the differences in the resistances of the ten sensors at each temperature are huge. This is most likely because the AOI process described previously was not performed in the $\mathrm{PCB}$ manufacturing process. In addition, the resistance measured at $55^{\circ} \mathrm{C}$ was subtracted from the resistance measured at $95^{\circ} \mathrm{C}$ to investigate the resistance variation rate in the temperature range used for DNA amplification during the PCR process. The mean of the differences, as a result of the subtraction, was $0.754 \Omega$. This number indicates that there is little variation in the 
resistance values. To investigate the degree of variation in detail, the mean, standard deviation, and coefficient of variation $(\mathrm{CV})$ of the resistance values for each sensor at set temperatures from $55^{\circ} \mathrm{C}$ to $95^{\circ} \mathrm{C}$ were calculated and verified

$\begin{aligned} & \text { Table 2. Mean, Standard Deviation, And Coefficient of Variatic } \\
& \text { Resistance Values at 55, }\end{aligned}$
\begin{tabular}{cccc} 
22, 85, and $\mathbf{9 5}^{\circ} \mathbf{C}$ \\
\hline Chip No & Mean & Std & CV \\
\hline 1 & 7.34 & 0.3551 & 4.84 \\
2 & 7.10 & 0.3423 & 4.82 \\
3 & 7.17 & 0.3474 & 4.85 \\
4 & 6.27 & 0.2980 & 4.75 \\
5 & 7.18 & 0.3500 & 4.87 \\
6 & 6.01 & 0.2861 & 4.76 \\
7 & 7.54 & 0.3714 & 4.93 \\
8 & 6.17 & 0.2938 & 4.76 \\
9 & 6.63 & 0.3160 & 4.77 \\
10 & 6.41 & 0.3025 & 4.72 \\
\hline
\end{tabular}

The CV values shown in Table 2 indicate that the degree of variation was small, at less than $5 \%$. Therefore, for the temperature difference between $55^{\circ} \mathrm{C}$ and $95^{\circ} \mathrm{C}$, i.e., $40^{\circ} \mathrm{C}$, the total variation of $0.754 \Omega$ corresponds to a predicted variation of $0.019 \Omega$ per ${ }^{\circ} \mathrm{C}$.

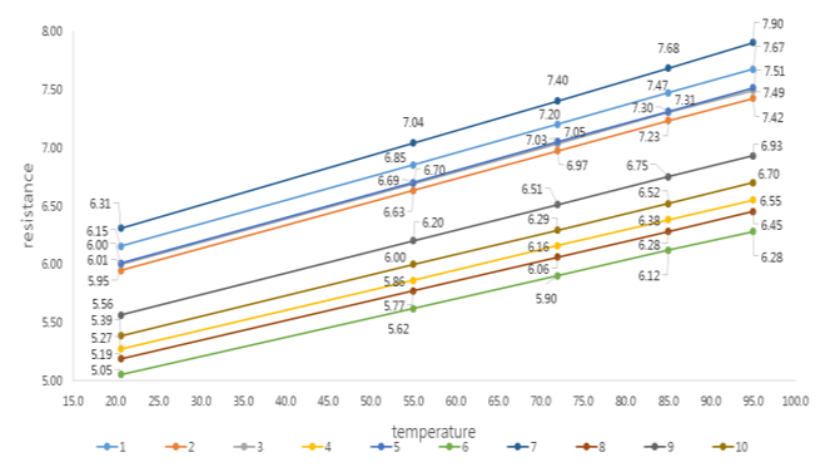

Figure 5. Resistance Values of PCB Patterns in Table 1 at Each Set Temperature

As Figure 6 shows, and as is typical of commonly used RTD sensors, the relationship between resistance and temperature is linear. The coefficient of correlation calculated for each of the ten sensors was greater than 0.99. In other words, the PCB-based RTD sensors used in this study behaved like commonly used RTD sensors.

\section{Conclusions}

In this study, RTD sensors were designed and fabricated using a PCB's $\mathrm{Cu}$ pattern for use as biochip temperature sensors. The experimental results showed that the difference in resistance was less than $1 \Omega$ for a temperature range from $55^{\circ} \mathrm{C}$ to $95^{\circ} \mathrm{C}$, and its $\mathrm{CV}$ varied by less than $5 \%$. These results indicate that the chip described in this paper is not suitable for use as a biochip temperature sensor, because the MCU cannot detect differences in resistance of less than $1 \Omega$ between $55^{\circ} \mathrm{C}$ to $95^{\circ} \mathrm{C}$. However, although the 
PCB-based RTD sensor is not suitable for use as a biochip temperature sensor, it exhibits potential for use as a general-purpose RTD sensor.

\section{Acknowledgments}

The research was supported by the Basic Science Research Program of the National Research Foundation of Korea (NRF), which is funded by the Ministry of Education, Science and Technology (2012R1A1A2040381) and by the Research and Business Development 2013 project of the Ministry of Trade, Industry and Energy (N0000907).

\section{References}

[1] J.M. Bartlett, D. Stirling, A short history of the polymerase chain reaction, in: PCR protocols, Springer, pp. 3-6 (2003).

[2] K.B. Mullis, H.A. Erlich, N. Arnheim, G.T. Horn, R.K. Saiki, S.J. Scharf, One of the first Polymerase Chain Reaction (PCR) patents, in, Google Patents, (1987).

[3] J.S. Hwang, S.Y. Kim, Y. S. Kim, H. J. Song, C. Y. Park, J. D. Kim, Implementation of PCB-Based PCR Chip Using Double-Sided Tape, International Journal of Control and Automation, (2015) Vol.8, pp.117

[4] J.-G. Lee, K.H. Cheong, N. Huh, S. Kim, J.-W. Choi, C. Ko, Microchip-based one step DNA extraction and real-time PCR in one chamber for rapid pathogen identification, Lab on a Chip, (2006), Vol.6, pp. 886-895.

[5] E. Salm, Y.-S. Liu, D. Marchwiany, D. Morisette, Y. He, A.K. Bhunia, R. Bashir, Electrical detection of dsDNA and polymerase chain reaction amplification, Biomedical microdevices, (2011), Vol. 13, pp.973982.

[6] J. Wu, R. Kodzius, K. Xiao, J. Qin, W. Wen, Fast detection of genetic information by an optimized PCR in an interchangeable chip, Biomedical microdevices, (2012), Vol. 14, 179-186.

[7] C. Koo, M. Malapi-Wight, H.S. Kim, O.S. Cifci, V.L. Vaughn-Diaz, B. Ma, S. Kim, H. Abdel-Raziq, K. Ong, Y.-K. Jo, Development of a Real-Time Microchip PCR System for Portable Plant Disease Diagnosis, PloS one, 8, e82704, (2013).

[8] C.-Y. Park, J.-D. Kim, J.-H. Ku, Y.-S. Kim, H.-J. Song, J. Kim, Printed Circuit Board-Based Polymerase Chain Reaction Chip, Sensor Letters, (2012), Vol. 10, pp. 1197-1202

[9] NATIONAL INSTRUMENTS http://www.ni.com/tutorial/7115/ko/

\section{Authors}

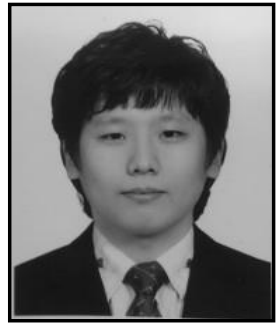

Sang-Yoon Kim received a master's degree in Computer Engineering from Hallym University. He is currently pursuing a Ph.D. in Computer Engineering at Hallym University. His research interests are in the areas of Bio-IT convergence and embedded systems.

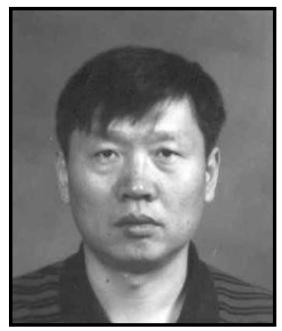

Jong-Dae Kim received M.S. and Ph.D. degrees in Electrical Engineering from the Korea Advanced Institute of Science and Technology in Seoul, Korea in 1984 and 1990, respectively. He worked for Samsung Electronics from 1988 to 2000 as an electrical engineer. $\mathrm{He}$ is a Professor in the Department of Ubiquitous Computing at Hallym University. His recent research has been focused on biomedical systems and bioinformatics. 


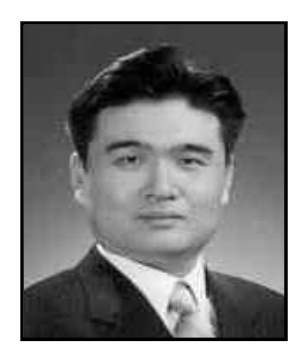

Yu-Seop Kim received a Ph.D. degree in Computer Engineering from Seoul National University. He is currently a Professor in the Department of Ubiquitous Computing at Hallym University. His research interests are in the areas of bioinformatics, computational intelligence, and natural language processing.

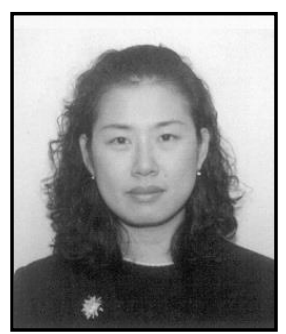

Hye-Jeong Song received a Ph.D. degree in Computer Engineering from Hallym University. She is a Professor in the Department of Ubiquitous Computing at Hallym University. Her recent research has been in the areas of biomedical systems and bioinformatics.

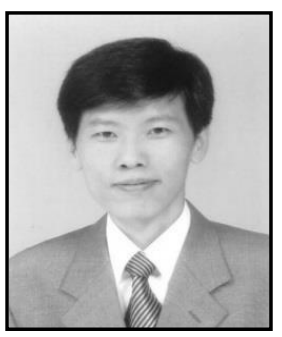

Chan-Young Park received a B.S. from Seoul National University and an M.S. and Ph.D. degree from the Korea Advanced Institute of Science and Technology in 1989 and 1995, respectively. From 1991 to 1999, he worked at Samsung Electronics. He is currently a Professor in the Department of Ubiquitous Computing of Hallym University. His research interests are in the areas of Bio-IT convergence, intelligent transportation systems, and sensor networks. 
International Journal of Control and Automation Vol.8, No.8 (2015) 